\title{
Les langues régionales au cœur des travaux sur la variation langagière : cent ans de publications de l'INRP
}

James Costa

\section{OpenEdition}

\section{Journals}

Édition électronique

URL : http://journals.openedition.org/trema/908

DOI : 10.4000/trema.908

ISSN : 2107-0997

Éditeur

Faculté d'Éducation de l'université de Montpellier

Édition imprimée

Date de publication : 1 septembre 2009

Pagination : 7-14

ISSN : 1167-315X

Référence électronique

James Costa, "Les langues régionales au cœur des travaux sur la variation langagière : cent ans de publications de l'INRP», Tréma [En ligne], 31 | 2009, mis en ligne le 01 septembre 2011, consulté le 20 avril 2019. URL : http://journals.openedition.org/trema/908 ; DOI : 10.4000/trema.908

Ce document a été généré automatiquement le 20 avril 2019

Trema 
Les langues régionales au cœur des travaux sur la variation langagière: cent ans de publications de l'INRP'

\author{
James Costa
}

I. Introduction 
1 La question des langues régionales provoque encore de nombreuses réticences en France aujourd'hui, bien qu'en ce qui concerne celles identifiées (par les services de l'État et par les militants) comme parlées sur le territoire métropolitain, elles soient en déclin et que leur usage, mis à part peut - être en Corse, au Pays Basque et en Alsace, soit résiduel. Ou peut - être justement à cause de cela.

2 L'argument généralement utilisé à leur encontre, en particulier dans les divers forums de discussion ouverts à la question par les journaux français à chaque fois qu'elle est d'actualité, est celui de l'inutilité de ces langues. Argument qui fait suite, historiquement, à celui de la menace à

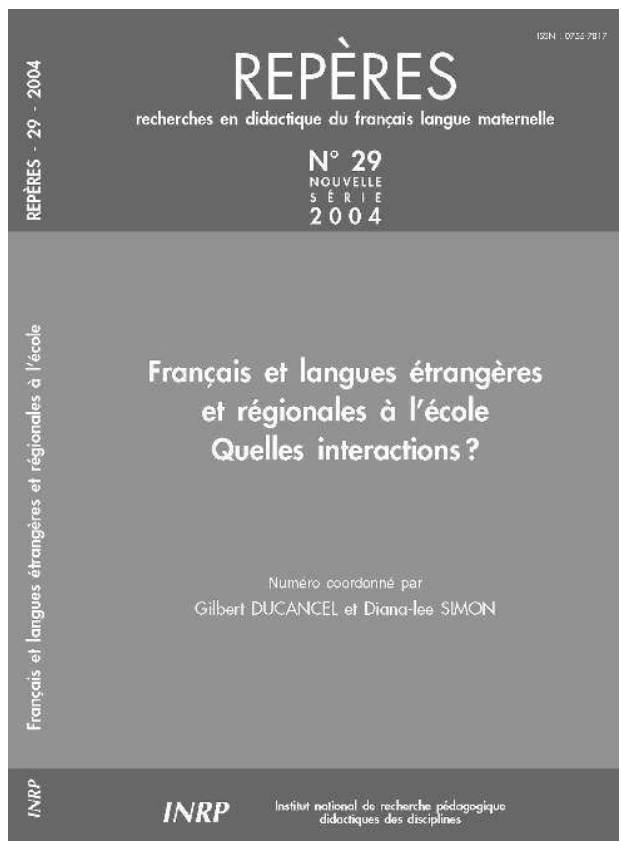
l'unité française et à la maîtrise de la langue française.

Ce sont, par ailleurs, les questions éducatives qui cristallisent les débats sur ces langues, tout au long du XXe siècle. En d'autres termes, les langues régionales, jusque dans les années 1950, n'ont pas leur place à l'école (mise à part la parenthèse pétainiste) parce qu'étant de toute manière parlées à l'extérieur de l'école, elles n'ont donc pas besoin d'être enseignées (c'est la variante politiquement correcte), et de plus, si elles l'étaient, elles nuiraient à l'enseignement du français (Martel, 2007). Après 1950, on considère qu'elles ne sont plus parlées ; comme elles ne constituaient plus une menace dans l'esprit du législateur, il était dès lors acceptable de leur concéder une petite place à l'école. C'est ce qui se produit en 1951 lorsque la Loi DEIXONNE est votée. Pourtant, dans l'esprit général, et si l'on en croit les divers commentaires politiques à partir de cette époque puis les données que nous pouvons lire dans les forums évoqués plus haut, ces langues sont désormais inutiles. À partir de cette argumentation, il en est déduit qu'il est donc inutile de consacrer une partie du temps scolaire, déjà chargé, à l'apprentissage de langues qui ne serviront pas hors du village des enfants, et qu'il vaut mieux leur enseigner l'anglais ou le chinois.

Ces quelques notes préliminaires montrent la prégnance des idéologies monolinguistes (et de fait nationalistes - cf. Lodge (2005, pp. 597 - 598) et plus généralement MILROY \& MILROY (1991 [1985])) qui transparaissent derrière ces quelques arguments fréquemment cités.

5 Nous montrerons ici, à travers un historique des travaux réalisés par l'INRP autour des questions des langues régionales, qu'il existe, et qu'il a toujours existé, d'autres voies permettant d'intégrer l'enseignement des langues régionales à un enseignement plus large qui traiterait la question linguistique, dans sa globalité. 


\section{Recherche en didactique et langues régionales : une histoire ancienne et un enterrement précoce}

Philippe MARTEL (2007 [1992]) a bien retracé l'histoire de l'attention qu'ont pu recevoir les langues régionales de France de la part des pédagogues du XIXe siècle, nous n'y reviendrons pas ici. Nous mentionnerons néanmoins le fait que les débats auxquels il se réfère ont lieu en grande partie dans la Revue pédagogique pendant les années 1890, revue fondée par Ferdinand BUISSON qui est également le fondateur du Musée Pédagogique, ancêtre de l'INRP, en 1879.

7 Si nous faisons ici référence à Ferdinand BUISSON, c'est qu'il est l'auteur d'un dictionnaire pédagogique de référence et qui connut deux éditions, la première en 1887, et la seconde en 1911. Si l'édition de 1887 fait une petite place aux 'patois' (MARTEL, 2007 [1992], p. 80), l'édition de 1911 y fait une seule brève allusion, à propos de la simplicité du vocabulaire des enfants et des erreurs de langage qu'ils seront amenés à commettre : «Dans les grandes villes, son langage naiff s'émaillera de termes empruntés à l'argot; dans les campagnes, c'est le patois ou les expressions locales qui viendront se mêler, ou se substituer, au français, indépendamment de l'accent qui, brochant sur le tout, variera avec la région ${ }^{2}$ "(nous soulignons). Cette contribution se trouve à l'article «langue maternelle ", que BUISSON estime être le français pour tous :

«Écoles primaires élémentaires. - On peut dire sans exagération de la langue maternelle qu'elle est le fond même de l'enseignement à l'école primaire. Elle domine et pénètre toutes les autres études; elle fournit par son seul objet le moyen pour chaque individu d'affirmer, de développer, de perfectionner sa personnalité. Grâce à la connaissance élémentaire, mais précise, qu'il en emporte en sortant de l'école primaire, l'élève est à même de comprendre et de goûter toutes les belles œuvres de notre patrimoine littéraire (...). ( (BUISSON, 1911).

8 On le voit, la langue maternelle est celle qui doit permettre de lire les classiques d'une littérature que l'on n'ose imaginer autre que française, même en Provence où Frédéric MISTRAL avait pourtant obtenu le Prix Nobel de littérature en 1904. Il est difficile de savoir si cette définition entérine une réalité en marche, à savoir la substitution linguistique bien entamée dans les diverses provinces françaises, ou s'il s'agit d'un effet particulièrement notable d'une idéologie française monolinguiste et hostile à toute forme de variation qui s'affirme de plus en plus. On notera que 1911 correspond à la date du dernier grand débat qui ait eu lieu autour de ces questions avant la Grande Guerre (MARTEL, 2007, p. 85 et suivantes), qui entérinera l'avancée du français tout en enterrant nombre des locuteurs des langues régionales de France, au sens figuré comme au sens propre.

9 Si des débats ont pu avoir lieu à la fin du XIXe siècle, voire au début du XXe, sur la place à accorder ou non aux idiomes locaux en tant qu'auxiliaires pour l'apprentissage de la langue nationale, ils ne sont plus réellement de mise après la guerre. Comme l'écrit Claude CORTIER (2008, p. 69) : «Le 14 août 1925, la circulaire MONZIE « sur les idiomes locaux » ôtait définitivement tout espoir aux partisans du comparatisme, en interdisant formellement, au nom de l'unité nationale, le recours aux «parlers régionaux » dans le cadre scolaire ».

10 La question des langues régionales ne devait plus faire l'objet de publications ou de débats particuliers dans les revues nationales traitant de pédagogie. La pédagogie des langues 
régionales devient l'apanage des militants dans les différentes régions françaises, et la création de l'Institut Pédagogique National (héritier du Musée Pédagogique et ancêtre direct de l'INRDP puis de l'INRP en 1976) en 1956, soit cinq ans après la Loi DEIXONNE, n'entraîne aucun changement à ce niveau.

\section{Question de la variation au cœur des préoccupations de l'INRP}

11 Il faut attendre la fin des années 1970 pour voir apparaître la question des langues régionales de nouveau dans une revue publiée par l'INRP, la revue Repères, en particulier à travers l'importance que prend la question de la variation, importance qui déclinera à la fin des années 1980 après l'émergence des didactiques des diverses disciplines.

12 Comme l'a écrit Claude CORTIER (2008, pp. 70 - 73), la question de la variation est au cœur de la réflexion de l'époque, la relation français / langues régionales étant reproblématisée dans le cadre d'une sociolinguistique scolaire (70). On notera en particulier les publications de FABRE et al. (1979), FABRE (1985), MARCELLESI (1985), LATERRASSE (1985), CADO et al. (1985), VARGAS (1988), LOBIER et FABRE (1992)3.

Il est intéressant de constater que ces articles et ces avancées sont publiés dans une revue consacrée explicitement au français langue maternelle, signe d'une didactique ouverte et faisant une place avant tout à la notion même de langage. Ainsi, « des collaborations vont s'installer entre linguistes, sociolinguistes, pédagogues et didacticiens, notamment au sein du groupe Varia de l'INRP tandis qu'à partir de 1982, les premières lois sur la régionalisation officialisent l'enseignement des «langues et cultures régionales » et le situent dans un ensemble cohérent de valeurs culturelles (Circulaire du 21 juin 1983)» (CORTIER, 2008, p. 70).

14 À cette époque, il s'agit donc de ne pas isoler les spécificités des langues régionales au sein de didactiques propres séparées des questions de langues maternelles (ce qu'elles sont encore pour certains élèves). La question de la variation est également très clairement posée à propos du français : le dogme de l'unicité de la langue nationale est donc remis en cause, et les formes régionales du français sont mises en avant. Il faut noter également la présence d'articles (FABRE, et al., 1979) écrits non seulement du point de vue de spécialistes du français mais également du point de vue de spécialistes des langues régionales. C'est l'occitan qui semble être le plus souvent mis en avant, du fait peut - être de sa proximité avec le français qui rend la création de ponts entre les deux langues non seulement bénéfique mais aussi indispensable, compte tenu de l'existence d'un interlecte fortement attesté, que BOYER $(1988,1990,2001)$ appelle « francitan ».

Ce type de travaux mettant en évidence «comment l'absence de "pesanteurs normatives » importantes (comme dans le cas du français) doit être conçue comme une chance pour la pédagogie et non comme un handicap » (CORTIER, 2008, p. 71) devait avoir des suites en Corse avec les approches polynomistes développées sur l'île en matière d'enseignement (mais voir JAFFE $(1999,2000)$ et JAFFE \& WALTON (2000) pour une appréciation critique), mais moins en pays d'oc par exemple, où les questions de norme cristallisent des questions idéologiques fortes, en particulier en Provence, ce qui a entrainé, en particulier depuis les années 2000, des crispations autour des questions de graphie et de bigraphisme (on lira - en parallèle - avec profit, à ce sujet, BLANCHET (2002) et SUMIEN (2006)). 
16 Par la suite, d'autres travaux ont suivi ceux du groupe Varia, et l'INRP publie deux numéros de Repères, en 1992 et en particulier en 2004 (où la question des langues régionales est abordée - cf. CORTIER \& DI MEGLIO (2004), seul article portant sur les langues régionales dans un numéro pourtant intitulé «Le Français et les langues régionales ou étrangères : quelles interactions?») mais comme le note Claude CORTIER (2008, p. 72) «la question du rapport à la Norme semble s'effacer devant les préoccupations d'ordre métalinguistique.

17 Ce numéro marque en effet l'entrée sur de devant de la scène en France des questions d'éveil aux langues avec un article de HAWKINS (1992). Un nouveau numéro, en 2008, comprend également un article sur les langues régionales et leur mise en convergence, didactiquement parlant, avec le français (CORTIER, 2008).

\section{La variation et les langues régionales, pour quoi faire?}

On voit ainsi que le travail de l'INRP autour des langues régionales, depuis leur prise en compte dans les années 1980 a surtout pour objet de les réintégrer au cœur d'une certaine convergence didactique avec des matières scolaires qui traitent des mêmes questions fondamentales au niveau linguistique (français, langues étrangères), voire plus récemment d'une didactique du plurilinguisme.

L'INRP mène ainsi, depuis 2002, des travaux sur les langues régionales, limités à la Corse pour commencer (cf. par exemple CORTIER \& DI MEGLIO, 2005, 2007) puis ouverts sur l'espace occitan à partir de 2006 (COSTA, 2007, 2008 ; COSTA \& CORTIER, 2009).

Ces dernières recherches s'inscrivent dans le cadre d'un travail sur l'étude des contacts de langues en milieu scolaire et sur la didactisation de ces contacts, dans une perspective qui allie sociolinguistique, anthropologie linguistique et écologie des langues (telle que définie par MÜHLHÄUSLER (1996, 2000), comme ensemble d'interactions structurées et faisant sens entre différentes langues - donc entre leurs locuteurs - parlées dans un même environnement).

21 Les langues régionales sont donc pleinement intégrées non seulement au travail de terrain effectué par l'INRP, qui collabore avec des enseignants d'école primaire et de collège à Marseille et à Orange, mais également à un travail de questionnement théorique sur les notions de plurilinguisme, d'hétérogénéité et de variation / variabilité linguistiques. Un travail est mené en parallèle sur des questions de revitalisation linguistique (cf. Costa, 2009 à paraître).

Enfin, l'INRP est impliqué dans un projet sous l'égide du Centre Européen pour les Langues Vivantes (CELV, situé à Graz) dans le cadre de son troisième appel à projets à moyen terme « Valoriser les professionnels en langues ». Le projet EBP - ICI (Éducation Bi / Plurilingue - Intercompréhension et Compétences Interculturelles) ${ }^{4}$

« vise à faire connaître, reconnaître, prendre en compte, didactiser dans le milieu scolaire et éducatif et au cœur de la classe les langues et variétés de langue présentes dans le contexte local / régional, les répertoires et pratiques langagières et le type d'interactions auxquelles elles peuvent donner lieu, tout en intégrant cependant la dimension patrimoniale. À ces matériaux élaborés, seront intégrés ceux des langues voisines ou étrangères, grâce aux réseau d'échanges d'enseignants et aux documents créés dans les autres régions » (CORTIER, 2009, pp. 114 - 115). 
Impliquant un certain nombre de contextes européens où l'on rencontre des situations de collatéralité au niveau linguistique (au sens où ELOY l'entend (ELOY, 2004; ELOY \& Ó. HIFEARNAIN, 2007)): Ecosse des Basses - Terres (scots et anglais), Val d'Aoste (francoprovençal, français, italien), Provence (provençal et français), Catalogne (catalan et français au nord, catalan et espagnol au sud). Le projet implique ainsi des partenaires en France (INRP), en Ecosse (Itchy - Coo), en Espagne (Universitat Autònoma de Barcelona) et en Italie (IRRE - VDA), chacun des partenaires travaillant à son tour avec un réseau d'enseignants qui contribuent au projet. En effet, pour citer Claude CORTIER, coordinatrice du projet,

«Si une approche en termes sociodidactiques s'avère pertinente sur ces terrains, elle implique aussi un rôle central pour les enseignants, comme nous avions pu le constater dans nos travaux sur le terrain corse. Ils sont médiateurs entre les acteurs en présence dans l'équipe pédagogique, mais aussi entre les chercheurs et les élèves et leur entourage. Leur engagement, leurs valeurs et leurs principes valorisent les pratiques et activités développées, rassurent enseignants, parents et institution. Ils sont également médiateurs social et didactique lorsqu'ils font entrer les langues et variétés minorisées dans la classe, c'est pourquoi il importe de les valoriser dans ces pratiques et de constituer des réseaux et des outils didactiques pour les aider à faire de ces langues et variétés des tremplins vers d'autres configurations plurilingues » (p. 115).

\section{Conclusion}

Les langues régionales de France, 65 ans après la Loi DEIXONNE, sont plus que jamais (et c'est sans doute leur dernière chance) dans une dynamique de revitalisation linguistique au sens le plus large du terme, c'est - à - dire de renversement de la dynamique de déclin pour aller vers une revernacularisation. Les travaux menés à l'INRP depuis plus de 30 ans montrent que si le chemin est encore long, s'engager dans des dynamiques qui isoleraient ces langues des autres disciplines linguistiques enseignées à l'école comme des pratiques quotidiennes auxquelles sont exposés les enfants ne mènerait qu'à accroître leur marginalisation.

C'est au contraire, nous semble - $t$ - $i l$, en les intégrant de manière croissante à l'économie des échanges quotidiens, pour paraphraser BOURDIEU, ainsi que, plus modestement, à des pratiques pédagogiques innovantes permettant de les situer socialement, historiquement autant que linguistiquement au sein d'ensembles plus vastes, leur conférant ainsi un sens qu'elles ont largement perdu, qu'elles pourront retrouver une signification sociale, condition essentielle à toute entreprise de revernacularisation. C'est ainsi également que l'école pourra donner un sens à ces langues pour tous ceux pour qui ces langues ne sont pas langues ancestrales, pour des raisons de migrations internes à l'hexagone ou de migrations internationales. Pour ceux également dont la langue a été langue de famille, c'est aujourd'hui souvent la génération des arrière - grands - parents qui a abandonné l'usage de la langue régionale. On peut concevoir que le sens de leur apprentissage n'apparaisse pas comme immédiat à leurs yeux.

S'il est certain que l'école ne peut à elle seule renverser une dynamique vieille d'au moins deux siècles (combien de bacheliers ont passé une langue régionale au baccalauréat ? Et combien d'entre eux ont transmis la langue à leurs enfants?), elle peut au moins contribuer à donner une vitalité nouvelle à ces apprentissages en recréant les ponts entre cultures et entre langues largement en contribuant à recréer des écologies linguistiques, 
intégrant l'ensemble des répertoires linguistiques des enfants, langues, accents, dialectes, variétés de la langue dominante. Quel meilleur cœur pour ce projet que l'enseignement de langues régionales qui sont, pour nombre d'entre elles, parlées dans plusieurs États européens et qui toutes, sont des portes vers d'autres langues et d'autres cultures?

\section{BIBLIOGRAPHIE}

BLANCHET, P. (2002). Langues, cultures et identités régionales en Provence : la métaphore de l'aïoli. Paris : L'Harmattan.

BOYER, H. (1988). Le « Francitan ». Matériaux pour l'approche des représentations et fonctionnements sociolinguistiques d'un interlecte. Lengas, $\mathrm{n}^{\circ} 23, \mathrm{pp}$. 71-96.

BOYER, H. (1990). Clés sociolinguistiques pour le « francitan ». Montpellier : CRDP de Montpellier.

BOYER, H. (2001). Le francitan, un « bâtard linguistique » qui a la vie dure. In H. BOYER \&P. GARDY (Eds.), Dix Siècles d'Usages et d'Images de l'Occitan - des Troubadours à Internet (pp. 415-430). Paris : L'Harmattan.

BUISSON, F. (1911). Dictionnaire de Pédagogie. Disponible en ligne : http :// www.inrp.fr/editionelectronique/lodel/dictionnaire-ferdinand-buisson/

CADO, S., HAMON, J., \& GARAUD, M. (1985). Apprendre le français en pays Gallo. Pourquoi prendre en compte le parler régional ? Repères, $\mathrm{n}^{\circ} 67$, pp. 71-78.

CORTIER, C. (2008). Français et langues régionales et/ou minoritaires : une mise en convergence difficultueuse. Repères $n^{\circ} 38$, pp. 63-80.

CORTIER, C. (2009). Propositions sociodidactiques face à la diversité des contextes. Vers une didactique convergente des langues collatérales et de proximité : éducation bi/plurilingue et projets interlinguistiques. Synergies Italie $\mathrm{n}^{\circ} 5$, pp. 109-118.

CORTIER, C. \& DI MEGLIO, A. (2004). Le dépassement du conflit diglossique en Corse : implications pédagogiques et didactiques chez les maîtres bilingues de l'école primaire. Repères, $n^{\circ} 29$, pp. 185-206.

CORTIER, C. \& Di MEGLIO, A. (2005). Modalités de scolarisation des enfants migrants, rapport à l'autre et construction du plurilinguisme dans les contextes d'enseignement bilingue français et langues régionales. In C. VAN DEN AVENNE (Ed.), Mobilités et contacts des langues, pp. 291-309. Paris : L'Harmattan.

CORTIER, C. \& DI MEGLIO, A. (2007). Pédagogie interactive, gestion plurilingue et rapport à la norme dans les écoles bilingues français - corse. In Auzaneau (Ed.), La mise en œuvre des langues dans l'interaction. Paris : L'Harmattan.

COSTA, J. (2007). La presse en langue régionale, un objet social à didactiser : le cas de la presse en provençal à partir d'une étude de cas, le mensuel Aquò d'Aquí In C. ALEN GARABATO (Ed.), Les langues de France au XXIe siècle : vitalité sociolinguistique et dynamiques culturelles. Paris : L'Harmattan.

COSTA, J. (2008). Langues et cultures régionales, langues vivantes étrangères. Quels enjeux pour une convergence didactique ? Cahiers de l'Acedle, 5(1), pp. 109-128.

COSTA, J. (2009 à paraître). Introduction à la revitalisation linguistique en Europe. In GRINEVALD, C. \& BERT, M. (Eds.), Travail de terrain sur langues en danger : locuteurs et linguistes. Paris : Ophrys. 
COSTA, J. \& CORTIER, C. (2009). Contacts de langues en milieu scolaire et développement du plurilinguisme. In I. PIEROSAK \& J.-M. ELOY (Eds.), Intervenir : appliquer, s'impliquer ? pp. 233-239. Paris : L'Harmattan.

ELOY, J.-M. (2004). Des langues collatérales : problèmes et propositions. In J.-M. ELOY (Ed.), Des langues collatérales: Problèmes linguistiques, sociolinguistiques et glottopolitiques de la proximité linguistique. vol. 1, pp. 5-25. Paris : L'Harmattan.

ELOY, J.-M. \& Ó HIFEARNAIN, T. (Eds.). (2007). Langues proches - langues collatérales; Near Languages - Collateral Languages. Paris : L'Harmattan.

FABRE, S. (1985). Approche de la représentation que les enfants se font de la langue régionale. Repères, ${ }^{\circ} 67$, pp. 59-70.

FABRE, S., Chabert, M.-H., \& Gros, G. (1979). L'occitan à l'école - Eveil aux parlers régionaux. Repères, $n^{\circ} 53$, pp. 28-87.

HAWKINS, E. (1992). La réflexion sur le langage comme « matière - pont » dans le programme scolaire. Repères, $\mathrm{n}^{\circ} 6$ (Nouvelle série), pp. 41-56.

JAFFE, A. (1999). Ideologies in Action : Language Politics on Corsica. Berlin \& New York : Mouton de Gruyter.

JAFFE, A. (2000). Introduction: Non-standard orthography and non-standard speech. Journal of Sociolinguistics, 4(4), pp. 497-513.

JAFFE, A. \& WALTON, S. (2000). The voices people read: Orthography and the representation of non-standard speech. Journal of Sociolinguistics, 4(4), pp. 561-587.

LATERRASSE, G. (1985). Pour une problématique de la reconnaissance des différences à l'école. Repères, $\mathrm{n}^{\circ} 67$, pp. 15 - 22.

LOBIER, A. \& FABRE, S. (1992). L'occitan au service des apprentissages linguistiques au cycle 2 de l'école primaire. Repères, ${ }^{\circ} 6$ (Nouvelle Série), pp. 139 - 154.

LODGE, R. A. (2005). Le clivage oc - oïl au moyen âge : Fiction méthodologique. Mélanges de l'École française de Rome. Moyen Age, 117(2), pp. 595 - 613.

MARCELLESI, C. (1985). Vers des pratiques pédagogiques plurinormalistes. Repères, n67, pp. 3-8.

Martel, P. (2007). L'école française et l'occitan - Le sourd et le bègue (Vol. 2). Montpellier : Presses Universitaires de la Méditerranée.

MARTEL, P. (2007 [1992]). Les pédagogues et les « patois » sous la Troisième République. In P. Martel (Ed.), L'école française et l'occitan : le sourd et le bègue (pp. 69-84). Montpellier : Presses de la Méditerranée.

MILROY, J. \& MILROY, L. (1991 [1985]). Authority in language (2 ed.). London \& New York: Routledge.

MÜHLHÄUSLER, P. (1996). Linguistic Ecology. London \& New York: Routledge.

MÜHLHÄUSLER, P. (2000). Language Planning and Language Ecology. Current Issued in Language Planning, 1(3), pp. 306-367.

SUMIEN, D. (2006). La standardisation pluricentrique de l'occitan : nouvel enjeu sociolinguistique développement du lexique et de la morphologie. Turnhout [Belgium/Belgique] : Brepols.

VARGAS, C. (1988). Construire la notion de variation langagière. Repères $n^{\circ} 76$, pp. 1-10. 


\section{NOTES}

1. Institut National de Recherche Pédagogique.

2. http://www.inrp.fr/edition-electronique/lodel/dictionnaire-ferdinandbuisson/

document.php ?id =3017

3. Il est à noter que l'ensemble des archives de la revue Repères est disponible en ligne sur le site de l'INRP, http://www.inrp.fr

4. http://ebp-ici.ecml.at

\section{RÉSUMÉS}

Cet article vise à faire le point sur 100 ans de travaux effectués par ou dans le sillage de l'INRP sur la question des langues régionales. Les contributions sont particulièrement importantes à partir des années 1980, où les langues régionales sont intégrées à des travaux sur la variation langagière qui associe français, langues régionales puis langues étrangères. Nous montrons ici l'intérêt de ces travaux aujourd'hui alors que, 65 ans après la Loi DEIXONNE, la place des langues régionales à l'école n'est toujours pas complètement légitime, et qu'avec la disparition annoncée des derniers locuteurs de la plupart d'entre elles, la question du sens de ces enseignements va se poser de manière forte.

In this article, we wish to assess the role of the INRP in the ongoing research about minority languages in France. Contributions are particularly significant as of the 1980s with works being conducted on linguistic variation. Minority languages are thus integrated in a larger scheme with French and, later, foreign languages. We would like to point the relevance of those works in contemporary France where, 65 years after the Loi Deixonne, regional minority languages are still not completely felt as legitimate in schools. With the announced decline and eventual death of most of these languages, it seems important to re - assess their role in education in order for them to remain meaningful in the eyes of an increasingly changing student population.

\section{INDEX}

Mots-clés : anthropologie linguistique, écolinguistique, INRP, langue régionale, variation

Keywords : ecolinguistic, linguistic anthropology, regional minority language

\section{AUTEUR}

JAMES COSTA

ICAR (CNRS UMR 5191, INRP et Université de Lyon) 